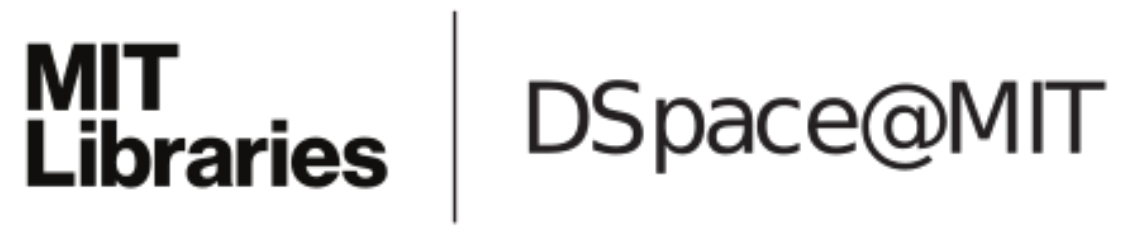

\author{
MIT Open Access Articles
}

Vertical Glider Robots for Subsea Equipment Delivery

The MIT Faculty has made this article openly available. Please share how this access benefits you. Your story matters.

Citation: Reed, Brooks L. et al. "Vertical glider robots for subsea equipment delivery." Proceedings of the 2011 IEEE International Conference on Robotics and Automation (ICRA) 109.39 (2011): 2356-2361.

As Published: http://ieeexplore.ieee.org/xpls/abs_all.jsp?arnumber=5980486

Publisher: Institute of Electrical and Electronics Engineers (IEEE)

Persistent URL: http://hdl.handle.net/1721.1/78655

Version: Author's final manuscript: final author's manuscript post peer review, without publisher's formatting or copy editing

Terms of use: Creative Commons Attribution-Noncommercial-Share Alike 3.0 


\title{
Vertical Glider Robots for Subsea Equipment Delivery
}

\author{
Brooks Reed, Charles Ambler, Julio Guerrero and Franz Hover
}

\begin{abstract}
We have developed an underwater vehicle that offers significant performance improvements over existing subsea elevators. Our Vertical Glider Robot falls under its own weight to a precise location on the seafloor, employing streamlining, active steering, and basic navigation instrumentation. We examine typical at-sea mission requirements, mention several key governing parameters, and outline our design approach. We then describe a prototype device, and present results from model-scale experiments.
\end{abstract}

\section{INTRODUCTION}

Among the various tasks performed by autonomous systems today in the deep ocean is delivery of equipment to specific locations on the seafloor. Such equipment includes scientific sensors [1], [2], acoustic network nodes [3], collection baskets [4], [5], underwater observatory docking equipment [6], military items, and others. Subsea delivery is achieved with powered underwater vehicles (autonomous underwater or remotely-operated vehicles; AUV's or ROV's) [7], [8] or unguided platforms (landers) [5], [9], [10], which fall passively. Powered vehicles can accomplish precision delivery with high performance because they can make repeated attempts to reach a given specification. But capital and operating costs of these vehicles can be orders of magnitude larger than the cost of the sensor being deployed; in the case of many packages to be delivered, these costs and the risk to major assets may be too high.

Oceanographic researchers and the offshore oil and gas industry regularly use passively dropped landers to deploy sensors to full ocean depth of up to six kilometers. This is achieved by positioning the surface vessel so that predicted ocean currents cause the lander to free-fall to the desired target. Over the length of the drop, these landers accumulate significant drift; $1 \%$ of depth is a typical value reported in deep water when a good current measurement is made $a$ priori. Due to drift, passive landers sometimes have to be recovered so that another attempt can be made. We note that in oil exploration, operating costs of the support vessel can be up to $\$ 500 \mathrm{~K}$ per day, so precise and timely delivery of equipment is important. As a particular application, the offshore industry would like to deploy sensors in a large grid

B. Reed is a graduate student in the Massachusetts Institute of Technology/Woods Hole Oceanographic Institution Joint Program in Oceanographic Engineering, Cambridge, MA 02139 USA. brooksr8@mit.edu

C. Ambler is a graduate of the Department of Mechanical Engineering at MIT, Cambridge, MA 02139 USA. charles.amblerealum.mit.edu

$\mathrm{J}$. Guerrero is a Lecturer with the Department of Mechanical Engineering at MIT, Cambridge MA 02138 USA. jguerrero1000@gmail.com www. linkedin.com/in/julioguerrero

F. Hover is an Assistant Professor with the Department of Mechanical Engineering at MIT, Cambridge, MA 02139 USA. hover@mit. edu on the seafloor, as shown in Fig. 1. To reduce ship costs, it is desired to allow all of the landers to be deployed from a single ship location near the center of the grid, motivating the need for horizontal transit capabilities.

Lander vehicles called elevators have been used for years to support ROV operations [5], and there has been some prior work on steerable elevators at the Woods Hole Oceanographic Institution (D. Yoerger and A. Bradley, personal communication). These elevators consisted of passive elevator frames retrofitted with wings, which spiraled down in a helix trajectory and could be steered manually in a rough manner via a single rudder and an acoustic link to the surface ship. While this project was sidelined in the early 2000's, there has been some recent work with model tests of steerable elevators [11]. Our objective in this paper is to propose a vehicle concept that improves on these steerable elevators by incorporating aspects from AUV and ROV operations and horizontal gliders [12]-[14]. To keep cost and complexity low, we retain the free-falling lander concept that uses potential energy instead of a powered propulsion system. Building on the steerable elevator concept, we propose to add fully autonomous navigation and active control, and to streamline the vehicle in order to add horizontal transit capabilities as well as reduce the large drift forces from largescale hydrodynamic separation. To distinguish our work from existing elevators and gliders as used in the ocean today, we refer to our device as the Vertical Glider Robot, or VGR.

Consistent navigation in the underwater environment is a perennial challenge because of our reliance on the acoustic channel [15]. Long baseline (LBL) acoustic systems can be integrated with an inertial measurement unit (IMU), depth, magnetic heading, and Doppler velocimetry (DVL) along a solid boundary to provide navigation accuracy on the order of one meter, but can be time-consuming to deploy [16]-[19] In contrast, ultra-short baseline (USBL) or short-baseline (SBL) systems [20] can be mounted on a ship and probably represent the easiest single means for maintaining global reference [21]. One aspect of USBL and SBL systems that demands special attention is their angular error characteristic, which will lead to a linear increase of noise on the Cartesian space estimate. Additionally, as with LBL, position updates are delayed many seconds as components move apart; the speed of sound in water is around $1450 \mathrm{~m} / \mathrm{s}$. For our Vertical Glider mission, we focus on a sensing mode akin to a shipmounted USBL, with depth, heading, and attitude measured onboard the vehicle.

The Vertical Glider is designed to have its principal orientation nose-down, with negative buoyancy to provide a constant dive rate. Most crucially, the vehicle is marginally 


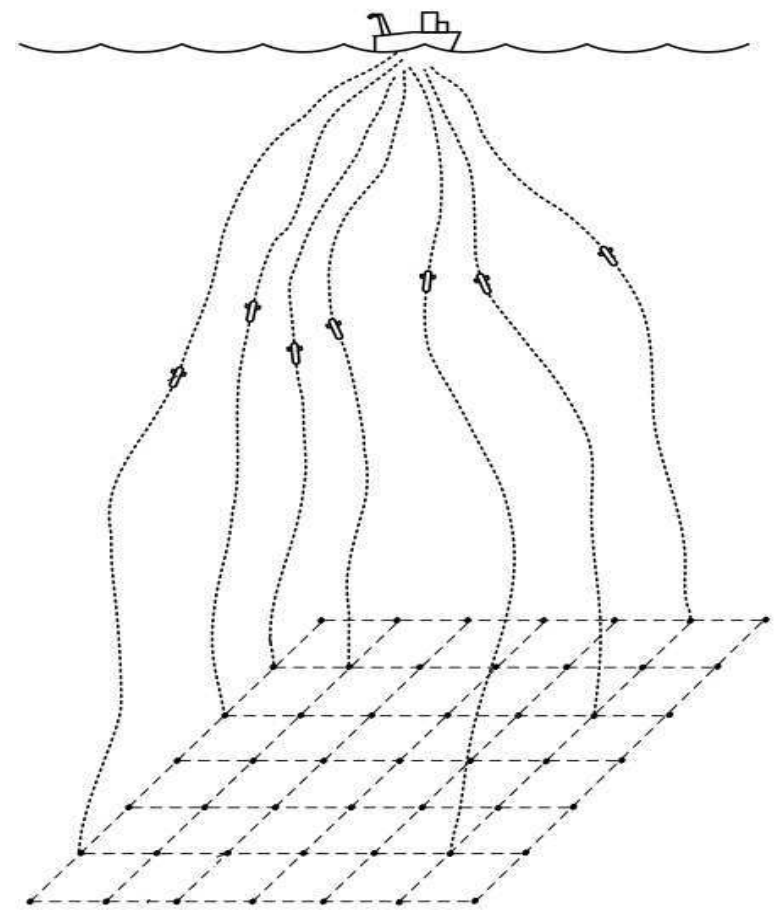

Fig. 1. Overview of a Vertical Glider mission scenario to deploy equipment on a grid on the seafloor from a single ship on the surface.

stable in the open loop, allowing it to operate at extreme angles of attack and thereby move at glide angles greater than 60 degrees from vertical, satisfying the need for moderate horizontal transit capability. High angle-of-attack flight will allow us also to modulate the vertical descent rate so as to more effectively share the limited USBL resource when multiple vehicles are deployed simultaneously. We describe the unique aspects of this type of vehicle and mission, and the design, control, and testing of a prototype vehicle.

\section{Prototype Vehicle Design}

\section{A. Physical Vehicle Design}

A prototype vehicle has been built to explore the behavior of vertically-oriented streamlined vehicles, including the effectiveness of control fins and achievable glide slopes. The vehicle has a simple, streamlined shape with control fins at the tail in the traditional cross configuration, as shown in Fig. 2. Table I lists some of the vehicle's physical characteristics. For control and data logging purposes, the vehicle's sensor suite includes an onboard tilt-compensated compass, pitch, and roll sensor (Ocean Server OS5000), a pressure sensor used to measure depth (Measurement Specialties M86), and angular rate gyros (Invensense IDG1250). An Arduino Mega microcontroller is used to read in sensors, compute control commands, drive servos and log data. Onboard data logging is handled by a $4 \mathrm{D}$ systems $\mu$ Drive microSD data logger. We use the CMUCam3 camera system for global navigation.

\section{B. Navigation Methods}

A camera tracking system is used in pool testing to emulate angle-based tracking methods used in the ocean.
TABLE I

Vertical Glider Physical PARAMETERS

\begin{tabular}{|r|l|}
\hline Length & $77 \mathrm{~cm}$ \\
\hline Diameter & $12.7 \mathrm{~cm}$ body, $30 \mathrm{~cm}$ at tips of fins \\
\hline Volume & $8040 \mathrm{~cm}^{3}$ \\
\hline Weight & $8.05 \mathrm{~kg}$ \\
\hline Weight in Water & $98 \mathrm{~g}$ \\
\hline Fin Profile & $\mathrm{NACA}-0020$ \\
\hline Design Dive Rate & $55 \mathrm{~cm} / \mathrm{s}$ \\
\hline Max Depth & $5 \mathrm{~meters}$ \\
\hline Servos & HiTec HS-322HD (x2) \\
\hline Power Source & $8 \times A A$ NiMH batteries $(1.2 \mathrm{~V}$ each, 9.6 V total) \\
\hline
\end{tabular}

Two major modes of operation using a camera are possible. One mode consists of the camera mounted in the nose of the vehicle. A flashlight is placed on the bottom of the pool to serve as the target; the camera tracks the light and the control system guides the vehicle towards the target. This method is different than the proposed surface ship navigation using a USBL, but has obvious applications in missions such as docking or homing towards an existing target [6], [22]. This capability is completely self-contained within the vehicle.

The second mode of operation matches deployment with a USBL on a ship more closely. A light is placed on the tail of the vehicle, and a surface raft holds a camera that tracks the light. The error in vehicle position is computed on a connected laptop at the surface, and this is combined with heading and attitude information received from the vehicle through a $2 \mathrm{~mm}$ diameter tether to compute commands for the vehicle's control surfaces. Matlab software is used for communication, control and logging on the laptop.

\section{Nondimensional Parameters}

Outlining several key nondimensional parameters should be useful to help us understand the scaling effects between different real-world missions and the pool tests. First, we note that the dynamic effects of lift, drag and inertia occur at much faster time scales than the basic mission parameters. The vehicle will have settled into a steady-state orientation by the time a new navigation update arrives, thus we assume that in deep water, the vehicle behaves as a kinematic particle, diagrammed in Fig. 3. The most relevant kinematic, noise and control parameters are listed in Table II; for more detailed simulation and control analysis, a more complex dynamic model could be used. The simple kinematic model analyzes motion in the $x-z$ plane: the lateral velocity of the vehicle in a global reference frame, $\dot{x}_{g}$, scales as the product of Cartesian position error and vertical velocity, i.e., $\dot{x}_{g}=K\left(\alpha_{\text {des }}-\alpha\right) \dot{z}$, with $\dot{z}$ constant. The main metric of system performance is the standard deviation of the landing error $\sigma_{E}$, given by:

$$
\sigma_{E}=f\left(K, \sigma_{\alpha}, \sigma_{w}, \dot{z}, D, c\right)
$$

This leads to a set of four nondimensional parameters for the nondimensional error [23] (note that $K$ and $\sigma_{\alpha}$ are already unitless): 

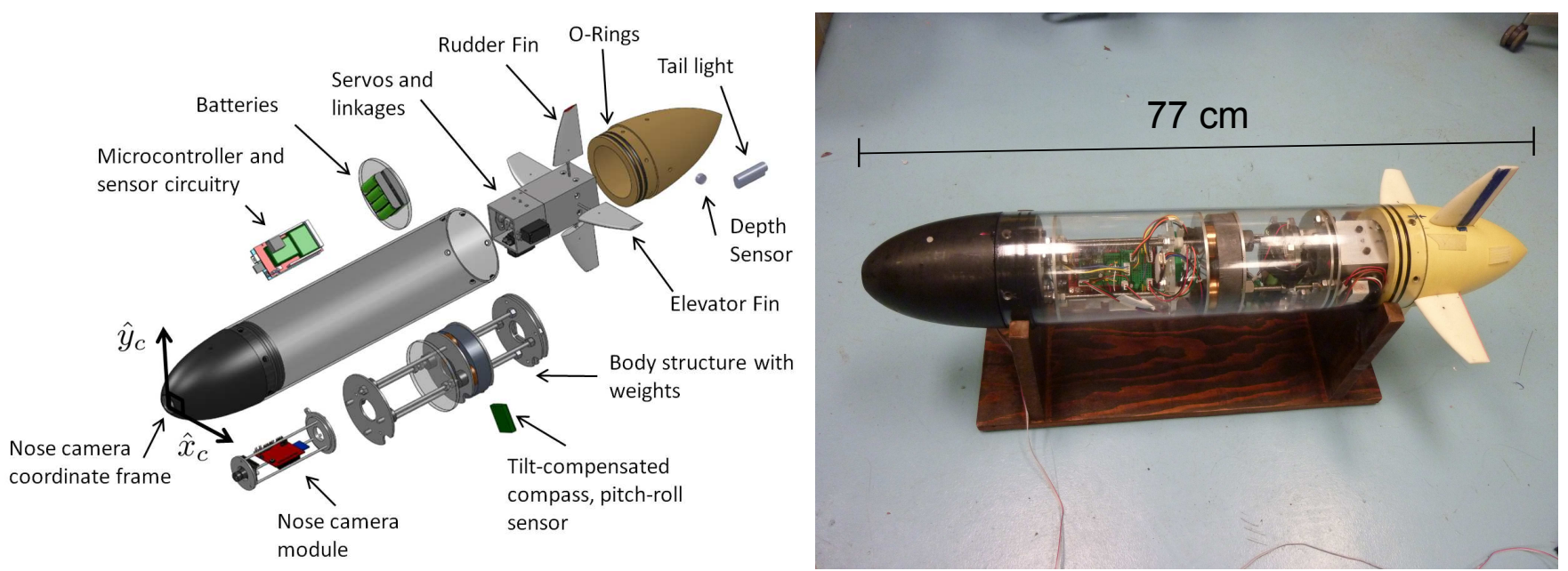

Fig. 2. An exploded view of the vehicle is on the left, including the onboard camera reference frame $\hat{x}_{c}$ and $\hat{y}_{c}$. A photograph of the vehicle with the communication tether attached to the side of the nose is on the right. Note the large lead weight near the center of the vehicle which was located to place the center of mass very slightly below the center of buoyancy, resulting in a marginally stable vehicle that can fly at high angles of attack.

TABLE II

Relevant Variables for Dimensional Analysis, Basic Kinematic Model

\begin{tabular}{|c|c|c|}
\hline Variable & Description & Units \\
\hline$\sigma_{E}$ & Std. Dev. of Landing Error & $\mathrm{m}$ \\
\hline $\mathrm{K}$ & Control Gain & $1 / \mathrm{rad}$ \\
\hline$\sigma_{\alpha}$ & Measurement Noise & $\mathrm{rad}$ \\
\hline$\sigma_{w}$ & Process Noise & $\mathrm{m} / \mathrm{s}$ \\
\hline$\dot{z}$ & Dive Rate & $\mathrm{m} / \mathrm{s}$ \\
\hline $\mathrm{D}$ & Mission Depth & $\mathrm{m}$ \\
\hline $\mathrm{c}$ & Speed of Sound in water & $\mathrm{m} / \mathrm{s}$ \\
\hline
\end{tabular}

$$
\frac{\sigma_{E}}{D}=f\left(K, \sigma_{\alpha}, \frac{\sigma_{w}}{\dot{z}}, \frac{\dot{z}}{c}\right)
$$

As written, the process noise is nondimensionalized with drop rate, and therefore characterizes the open-loop drift in lateral position relative to depth. The ratio $\dot{z} / c$ essentially scales the number of navigation hits that the vehicle will receive from the USBL. If the USBL system used is capable of ping stacking, navigation hits will arrive at a uniform $1 \mathrm{~Hz}$ update rate, so the scale of this parameter will change. While ping-stacking improves the update rate, it does not eliminate the delay between the USBL measurement of a vehicle and the time when the vehicle receives the measurement, which is constrained by $c$.

\section{PROTOTYPE VEHICLE MODELING AND CONTROL}

\section{A. Vehicle Modeling}

For modeling purposes, we consider motions in the vertical plane, independent of vehicle twist about its central axis. Lateral motion is induced by pitch, because of lift forces generated by the body at an angle of attack. Based on data obtained in step responses for pitch, a simple secondorder transfer function for the vehicle's pitch dynamics was derived. This relates the input elevator fin angle to the output

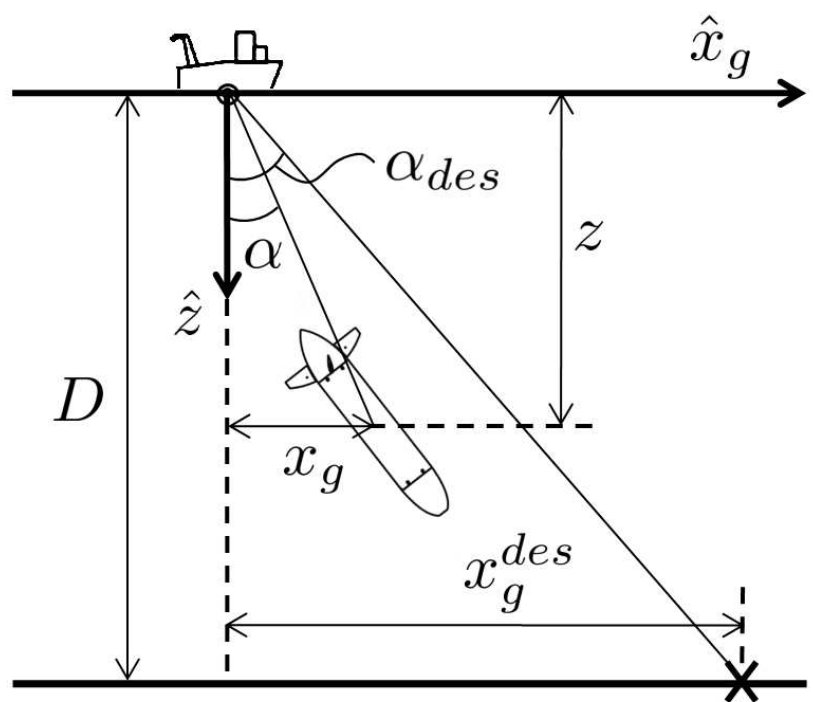

Fig. 3. Diagram for surface measurement and simple kinematic model, used for dimensional analysis only. In a $2 \mathrm{D}$ plane, the angle from the support vehicle to the target is $\alpha_{d e s}$, the angle from the support vessel to the vehicle is $\alpha$, full ocean depth is $D$, the vehicle's depth is $z$, the location of the target is $x_{g}^{\text {des }}$, and the vehicle's horizontal distance from the origin is $x_{g}$.

pitch angle of the vehicle at the vehicle's designed dive rate of $55 \mathrm{~cm} / \mathrm{s}$ :

$$
\frac{\text { Pitch }}{\text { Elevator }}=\frac{3.8}{s^{2}+2.88 s+0.81}
$$

The system has a damping ratio of 1.6, showing that the system is overdamped. The natural frequency is $0.9 \mathrm{rad} / \mathrm{s}$. We have observed visually over a number of tests that the steadystate pitch achievable by the vehicle is over 60 degrees; however, this linear model loses accuracy at high fin angles and high angle of attack. A more accurate model would include high angle effects due to nonlinear separation and 
body lift. Additionally, we note that a more accurate transfer function would include nonminimum phase aspects of the system, which are especially noticeable for the prototype measurement system that tracks a light on the tail.

\section{B. Flight Control with Onboard Camera}

One primary advantage to the onboard camera is that flight control is very simple because measurement, control and actuation are all kept in the vehicle body-referenced frame. No information about the vehicle's orientation is needed for the controller. The elevators correct for errors in the camera's $y$ axis, $\hat{y}_{c}$, and the rudders correct for errors in the $x$ axis, $\hat{x}_{c}$, as diagrammed in Fig. 2. A simple proportional controller maps the target location in the camera's field of view $-x_{c}$ and $y_{c}$, measured in pixels - to fin commands, attempting to keep the target in the center of the camera's field of view:

$$
\left\{\begin{array}{c}
\theta_{\text {elevator }} \\
\theta_{\text {rudder }}
\end{array}\right\}=\left\{\begin{array}{l}
-K y_{c} \\
-K x_{c}
\end{array}\right\}
$$

If the camera loses the target, the fins are both held at their previous position.

\section{Flight Control with Surface Camera}

The surface camera is located at the origin of a global North-East-Depth inertial coordinate frame, which is represented by $\hat{x}_{g}, \hat{y}_{g}$ and $\hat{z}_{g}$ in Fig. 4 . We use the depth of the vehicle, $z$, and the camera target pixel locations to find the tail location in global coordinates: $x_{g}$ and $y_{g}$. We subtract the target, $x_{g}^{d e s}$ and $y_{g}^{d e s}$, from the tail's location in the global frame to get a global horizontal-plane error vector, $e_{g}^{x}$ and $e_{g}^{y}$. The vehicle's body-referenced frame $\hat{x}_{v}, \hat{y}_{v}$ and $\hat{z}_{v}$ is aligned with $\hat{z}_{g}$ but is rotated in the horizontal plane by the vehicle's compass heading. The compass heading $\psi$ is the angle of rotation of the body-referenced frame from magnetic North (set to equal $\hat{x}_{g}$ in Fig. 4), which is computed onboard the tilt-compensated compass sensor using data from magnetometers and accelerometers on all three axes. We transform the global error vector into a vehicle body-referenced error, $e_{v}^{x}$ and $e_{v}^{y}$, through a rotation matrix that uses $\psi$ :

$$
\left\{\begin{array}{l}
e_{v}^{x} \\
e_{v}^{y}
\end{array}\right\}=\left[\begin{array}{cc}
\cos (\psi) & -\sin (\psi) \\
\sin (\psi) & \cos (\psi)
\end{array}\right]\left\{\begin{array}{c}
e_{g}^{x} \\
e_{g}^{y}
\end{array}\right\}
$$

Vehicle pitch is a rotation about the vehicle's bodyreferenced $x$ axis, $\hat{x}_{v}$, and is actuated by the elevators. Vehicle roll is a rotation about the vehicle's body-referenced $y$ axis, $\hat{y}_{v}$, and is actuated by the rudders. Using the depth of the pool, $D$, the vehicle's current depth, $z$, and the vehicle body-referenced errors, angles to the target about the vehicle's $x$ and $y$ axes, $\theta_{x}$ and $\theta_{y}$, are calculated:

$$
\left\{\begin{array}{l}
\theta_{x} \\
\theta_{y}
\end{array}\right\}=\left\{\begin{array}{l}
\operatorname{atan}\left(e_{v}^{y} /(D-z)\right) \\
\operatorname{atan}\left(e_{v}^{x} /(D-z)\right)
\end{array}\right\}
$$

Since the vehicle's pitch and roll dynamics are faster than its dynamics in the horizontal plane, a closed-loop pitch and roll controller commands the fins to angles $\theta_{\text {elevator }}$ and $\theta_{\text {rudder }}$

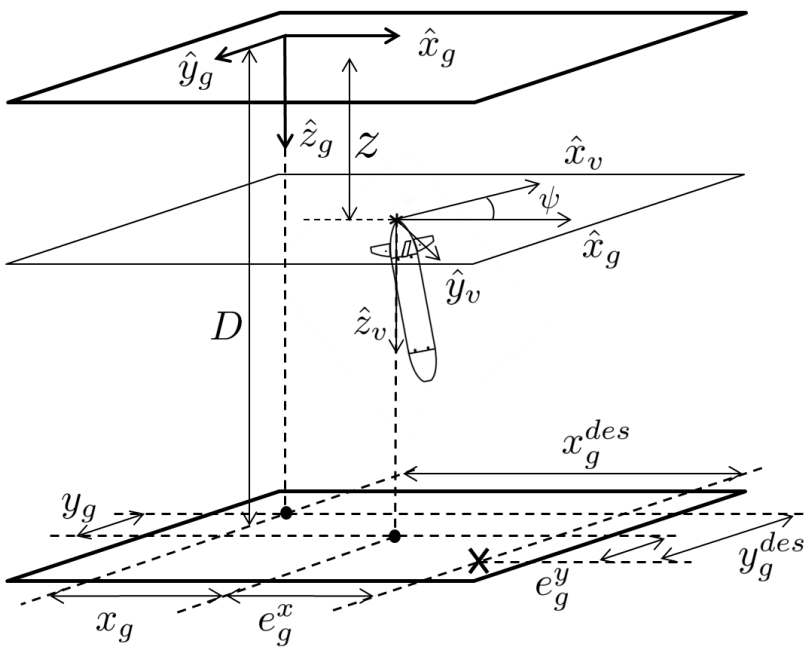

Fig. 4. 3D coordinate frames used for flight control with surface camera The global frame $\hat{x}_{g}, \hat{y}_{g}, \hat{z}_{g}$ is centered at the location of the surface raft. The body-referenced frame $\hat{x}_{v}, \hat{y}_{v}$ and $\hat{z}_{v}$ is aligned with $\hat{z}_{g}$ but is rotated in the horizontal plane by the vehicle's compass heading, $\psi$.

to attempt to drive the vehicle to the desired angle to the target, using proportional control with gain $K$ :

$$
\left\{\begin{array}{c}
\theta_{\text {elevator }} \\
\theta_{\text {rudder }}
\end{array}\right\}=\left\{\begin{array}{c}
-K\left(\text { Pitch }-\theta_{x}\right) \\
-K\left(\text { Roll }-\theta_{y}\right)
\end{array}\right\}
$$

\section{PROTOTYPE EXPERIMENTS IN POOL}

Testing was conducted in the MIT Alumni Pool (4m depth) and the MIT Z-Center Pool (4.25m depth).

\section{A. Onboard Camera}

We conducted several experimental runs to a flashlight target on the bottom of the swimming pool with the onboard camera configuration. A plot showing the camera's adjusted target over the course of a run is shown in Fig. 5. Starting from a variety of initial positions and angles, the vehicle hit the target within $25 \mathrm{~cm} 26$ times and veered off course due to loss of the target in the camera field of view 3 times. The times when it veered off track were due to testing the limits of extreme initial conditions. During these closed-loop tests, we noted the vehicle was able to reach targets that required a trajectory of 45 degrees from the launching point.

\section{B. Surface Camera}

To test the surface camera, we placed and surveyed a target on the bottom of the pool that was $3 \mathrm{ft}$ directly to the East of the surface camera. To show the vehicle's control capabilities, we started the vehicle in different orientations both the angles in the E-Z and N-Z planes and the rotation about the vehicle's axis. We observed some runs where the vehicle rotated a full 360 degrees about its primary axis, showing that our transformation from global to vehicle frame based on heading was working correctly.

Plots showing the vehicle's trajectory for three runs to the target with different initial conditions are shown in Figure 6. 


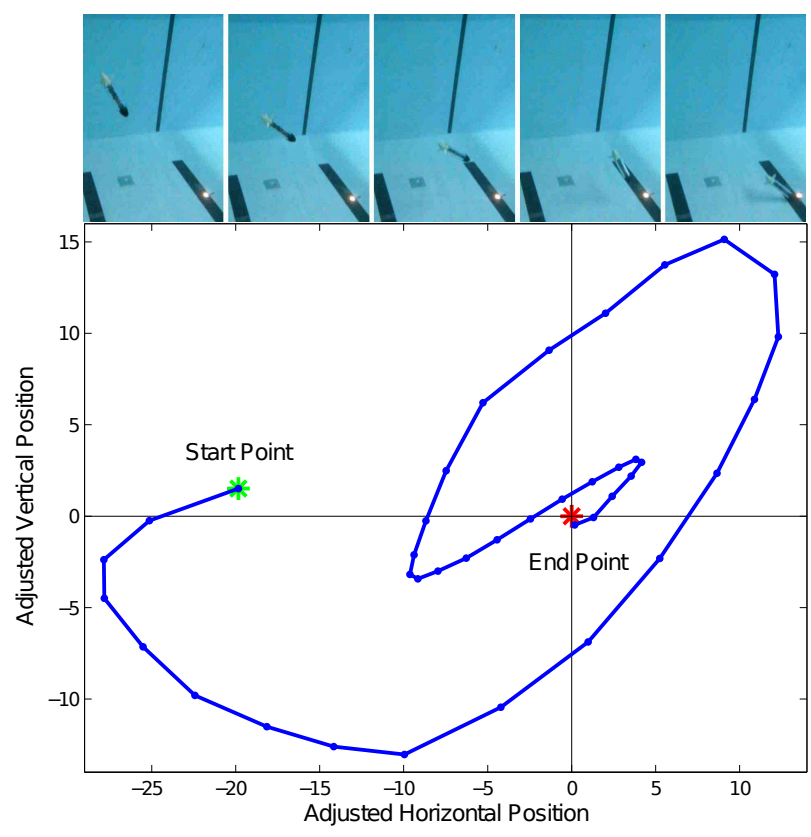

Fig. 5. Scatter plot showing the adjusted target location as seen by the vehicle's onboard camera during pool testing. For this plot, the camera's output in pixels is scaled by the radius of the target as seen by the camera, which adjusts for the angle-accentuating effects of the vehicle's distance to the target. The vehicle was launched from a point $3 \mathrm{~m}$ horizontally away from the target.

The vehicle corrects for drift in the $\mathrm{N}-\mathrm{Z}$ plane over the course of the run. The vehicle tracks the desired angle to the target in the E-Z plane well, but due to inaccuracies in the system and a simplified controller, it overshoots the target slightly, by an amount proportional to its initial angle towards the target.

One major limitation on this test was the camera's field of view. The CMUCam has a field of view of 49 degrees in $x$ and 37 degrees in $y$, which limits the 'cone' in which the vehicle can be seen by the camera. USBL systems in the ocean also have a limited cone of detection, due to attenuation of the signal to reduce noise from the ship machinery at shallow angles. While the CMUCam's field of view is a tighter constraint than typical USBL detection cones, we were able to learn about the effects of this constraint on operations through our testing. The limited cone means that we could not command the vehicle to go to targets very far away, and the margin for testing initial vehicle orientations was limited.

Additionally, the surface raft that holds the camera was designed to resist wave disturbances; however, some pitch and roll oscillations were observed that added noise onto the measurements. Adding a pitch and roll sensor to the raft could remove this noise, just as is done with a real USBL system on a ship. Regarding the control system, the vehicle had some backlash and calibration errors on the fins, which can add errors over a short drop. For the tests shown, the controller computes control actions based off the position of the light at the tail, not the vehicle's center of gravity (CG). This introduces angular error and accentuates
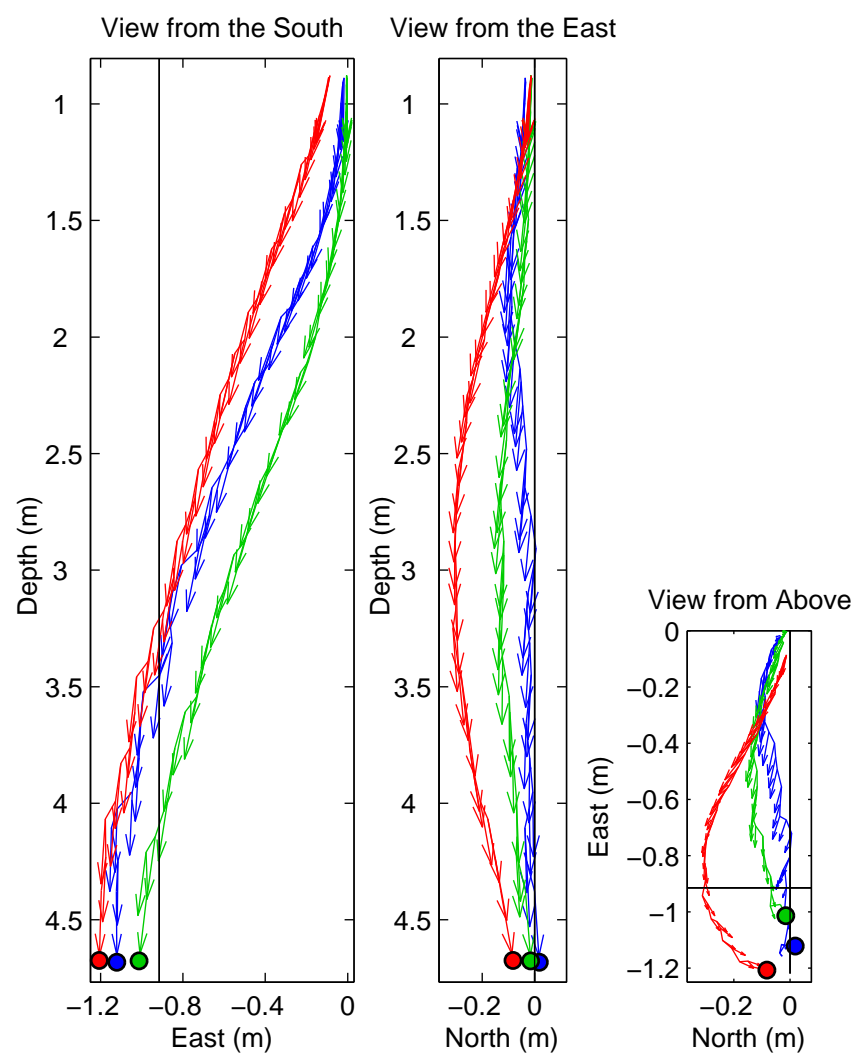

Fig. 6. Trajectory results from pool experiments. The target was $0.9 \mathrm{~m}$ directly to the West, as shown by the black lines.

nonminimum phase aspects of the measured system. An improved controller would account for the difference between the measurement and vehicle's CG and also attempt to drive the vehicle directly over the target first, and then drop straight down. These issues were ignored for our initial tests, and explain some of the overshoot observed in the results.

\section{CONCLUSIONS AND FUTURE WORKS}

We have identified significant improvements to be made over existing subsea equipment deployment methods, by adding fully autonomous navigation and active control to a more streamlined evolution of the steerable elevator concept. We have designed a suitable prototype vehicle and demonstrated proof-of-concept tests of closed-loop control. To keep cost and complexity low, our Vertical Glider Robot falls under the force of gravity alone with minimal sensors and actuators onboard, yet can be actively guided to achieve highaccuracy landing positions. While the vehicle nominally flies nose-downward, it can also cover horizontal distances on par with the water depth and can modulate its vertical speed. Our prototype vehicle has been tested with two navigation arrangements, both based on vision. We achieved good hit performance, within the limitations of pool testing.

In the future, it is likely that large groups of such vehicles will be deployed simultaneously, saving ship time and associated operating costs. To share the same navigation sensor 
among vehicles, e.g., individual USBL hits, we require a dynamic allocation policy to decide which vehicle needs the next measurement most. One structure that seems promising is the restless multi-armed bandit [24], [25]. We have already investigated an approximation of the bandit filtering problem given in [26]. Initial computations comparing the approximate bandit algorithms with round-robin, greedy, epsilongreedy, and random measurement schemes show mild but consistent benefits. An extension of this tracking problem could conceivably also control descent rate among vehicles, for the purpose of precisely hitting targets at the bottom.

\section{ACKNOWLEDGMENTS}

This work was supported by a grant from SchlumbergerDoll Research, Cambridge, MA. The authors would like to thank Roberto Melendez, Jasmine Florentine, Robert Hummel, Josh Leighton, Josh Taylor and Brendan Englot for help with pool testing; and Dana Yoerger and Al Bradley for discussions regarding the usage of elevator vehicles to support oceanographic research.

\section{REFERENCES}

[1] S. Constable, A. Orange, G. Hoversten, and H. Morrison, "Marine magnetotellurics for petroleum exploration, Part I: A sea-floor equipment system," Geophysics, vol. 63, no. 3, pp. 816-825, 1998.

[2] S. Bradley, S. Addison, I. Priede, M. Collins, and P. Bagley, "A deep-ocean fish tracking system using code-activated transponders and hydrophone array," in Electronic Engineering in Oceanography, 1997. Technology Transfer from Research to Industry., Seventh International Conference on, June 1997, pp. $34-38$.

[3] J. Heidemann, Y. Li, A. Syed, J. Willis, and W. Ye, "Underwater sensor networking: Research challenges and potential applications," in Proceedings of the IEEE Wireless Communications and Networking Conference, 2006.

[4] R. Ballard, L. Stager, D. Master, D. Yoerger, D. Mindell, L. Whitcomb, H. Singh, and D. Piechota, "Iron age shipwrecks in deep water off Ashkelon, Israel," American Journal of Archaelology, vol. 106, no. 2, pp. 151-168, 2002.

[5] M. Bowen, P. Bernard, D. Gleason, and L. Whitcomb, "Elevatorsautonomous transporters for deepsea benthic sample recovery," in OCEANS 2000 MTS/IEEE Conference and Exhibition, vol. 1, 2000, pp. $343-348$ vol.1.

[6] H. Singh, J. Catipovic, R. Eastwood, L. Freitag, H. Henriksen, F. Hover, D. Yoerger, J. Bellingham, and B. Moran, "An integrated approach to multiple AUV communications, navigation and docking," in OCEANS 1996 MTS/IEEE Conference Proceedings, vol. 1, Sep 1996, pp. 59-64 vol.1.

[7] L. Whitcomb, "Underwater robotics: out of the research laboratory and into the field," in IEEE International Conference on Robotics and Automation, 2000. Proceedings., vol. 1, 2000, pp. 709-716.

[8] F. Frisbie, R. Wernli, and D. Given, "The Role of the ROV in 1985: A Capability in Transition," in Offshore Technology Conference, 1986.
[9] I. Priede, S. Addison, S. Bradley, P. Bagley, P. Gray, C. Yau, J. Rolin, J. Blandin, J. Legrand, A. Cremer, U. Witte, O. Pfannkuche, A. Tengberg, S. Hulth, W. Helder, and T. Van Weering, "Autonomous deepocean lander vehicles; modular approaches to design and operation," in OCEANS 1998 MTS/IEEE Conference Proceedings, vol. 3, 1998, pp. $1238-1244$.

[10] J. Byron and R. Tyce, "Designing a Vertical / Horizontal AUV for Deep Ocean Sampling," in Oceans 2007 MTS/IEEE Conference Proceedings, 2007, pp. 1-10.

[11] C. Roman, T. Gregory, E. Martin, A. Sanguinetti, and J. Drummond, "Preliminary model tests for the design of a gliding deep water elevator," in OCEANS 2007, 292007-oct.4 2007, pp. 1 -5.

[12] J. Sherman, R. Davis, W. Owens, and J. Valdes, "The autonomous underwater glider 'Spray',' IEEE Journal of Oceanic Engineering, vol. 26, no. 4, pp. 437-446, Oct 2001.

[13] D. Webb, P. Simonetti, and C. Jones, "SLOCUM: an underwater glider propelled by environmental energy," IEEE Journal of Oceanic Engineering, vol. 26, no. 4, pp. 447-452, Oct 2001.

[14] C. Eriksen, T. Osse, R. Light, T. Wen, T. Lehman, P. Sabin, J. Ballard, and A. Chiodi, "Seaglider: a long-range autonomous underwater vehicle for oceanographic research," IEEE Journal of Oceanic Engineering, vol. 26, no. 4, pp. 424-436, Oct 2001.

[15] M. Stojanovic, "Recent advances in high-speed underwater acoustic communications," IEEE Journal of Oceanic Engineering, vol. 21, no. 2, pp. 125-136, 1996.

[16] L. L. Whitcomb, D. R. Yoerger, and H. Singh, "Combined Doppler/LBL based navigation of underwater vehicles," in Proceedings of the 11th International Symposium on Unmanned Untethered Submersible Technology, 1999.

[17] J. Kinsey, R. Eustice, and L. Whitcomb, "A Survey of Underwater Vehicle Navigation: Recent Advances and New Challenges," in IFAC Conference of Manoeuvering and Control of Marine Craft, Lisbon, Portugal, September 2006, invited paper.

[18] N. Kussat, C. Chadwell, and R. Zimmerman, "Absolute positioning of an autonomous underwater vehicle using GPS and acoustic measurements," IEEE Journal of Oceanic Engineering, vol. 30, no. 1, pp. 153-164, 2005.

[19] P. Rigby, O. Pizarro, and S. Williams, "Towards Geo-Referenced AUV Navigation Through Fusion of USBL and DVL Measurements," in OCEANS 2006 MTS/IEEE Conference Proceedings, Sep. 2006, pp. 1 $-6$.

[20] K. Vickery, "Acoustic positioning systems: A practical overview of current systems," in Proceedings Of The 1998 Workshop on Autonomous Underwater Vehicles, 1998., Aug 1998, pp. 5-17.

[21] "Kongsberg acoustic underwater positioning and navigation systems," Kongsberg Maritime. [Online]. Available: http://www.km.kongsberg.com

[22] R. Stokey, M. Purcell, N. Forrester, T. Austin, R. Goldsborough, B. Allen, and C. von Alt, "A docking system for REMUS, an autonomous underwater vehicle," in OCEANS 1997 MTS/IEEE Conference Proceedings, vol. 2, Oct 1997, pp. 1132-1136.

[23] C. Ambler, "Design of an Underwater Vertical Glider for Subsea Equipment Delivery," Master's thesis, Massachusetts Institute of Technology, 2010.

[24] J. Gittins, "Bandit Processes and Dynamic Allocation Indices," Journal of the Royal Statistical Society, Series B., vol. 41, no. 2, pp. 148-177, 1979.

[25] P. Whittle, "Restless Bandits: Activity Allocation in a Changing World," Journal of Applied Probability, vol. 25, pp. 287-298, 1988.

[26] J. Le Ny, Eric Feron, and Munther Dahleh, "Scheduling Kalman Filters in continuous time," Arxiv preprint: arXiv:0810.5148, 2008. 